\title{
Two-center experience with Neuroform Atlas stent-assisted coil occlusion of broad-based intracranial aneurysms
}

\author{
Gamze Arslan $^{1} \cdot$ Volker Maus $^{1} \cdot$ Werner Weber ${ }^{1} \cdot$ Ansgar Berlis $^{2} \cdot$ Christoph Maurer $^{2} \cdot$ Sebastian Fischer $^{1}$
}

\begin{abstract}
Purpose Stent-assisted coiling (SAC) represents an established treatment option for broad-based intracranial aneurysms. Here we report our initial and follow-up experience with the Neuroform Atlas Stent, a hybrid open- and closed-cell low-profile stent in the treatment of broad-based aneurysms.

Methods All intracranial aneurysms treated by SAC with the intention to apply the Neuroform Atlas Stent between July 2015 and December 2019 were included. Angiographic and clinical results were analyzed including all follow-up examinations.

Results A total of 119 aneurysms (8 acutely ruptured) in 112 patients were included. In 19 cases (16.0\%) recatheterization of the aneurysm was performed in a second procedure after failure to re-cross the stent initially. Of all aneurysms, $83.2 \%$ (99/119) were completely occluded following the procedure. In 75.6\% of all cases (90/119), a single microcatheter was used for both, implantation of the stent and coil occlusion of the aneurysm. At 3-6 and 12 months follow-up, the complete occlusion rates were 75.2 (79/105) and 81.3\% (74/91). The thromboembolic (stent thrombosis) and hemorrhagic complication rate was $1.7(2 / 119)$ and $0.8 \%(1 / 119)$, respectively, resulting in a procedure-related morbidity and mortality rate of 0.9 and $0.9 \%$ (1/112 patients).

Conclusion SAC using the Neuroform Atlas Stent offers a safe and effective option to treat broad-based intracranial aneurysms with a high angiographic and clinical success rate. In the majority of procedures, the application of two mircocatheters is not required. Prospective comparative studies might help to identify the value of SAC using the Neuroform Atlas Stent among the growing treatment options for broad-based aneurysms.
\end{abstract}

\section{Abbreviations}

SAC stent-assisted coiling

FDA Food and Drug Administration

$\mathrm{mRS}$

RROC modified Rankin score

A com

Sebastian Fischer

sebastian.fischer@kk-bochum.de

1 Knappschaftskrankenhaus Bochum - Universitätsklinik, Institut für Diagnostische und Interventionelle Radiologie, Neuroradiologie, Nuklearmedizin, In der Schornau 23-25, 44892 Bochum, Germany

2 Klinik für Diagnostische Radiologie und Neuroradiologie, Universitätsklinikum Augsburg, Augsburg, Germany
ACA anterior cerebral artery

ACA A1 pre-communicating segment of ACA

ACA A2 post-communicating segment of ACA

MCA bif middle cerebral artery bifurcation

$\mathrm{M} 1$

M2

BA tip

BA tr

PCA

PICA

ICA

ICA bif

ICA P com

proximal segment of the MCA

post bifurcation segment of the MCA

basilar artery tip

basilar artery trunk

posterior cerebral artery

posterior inferior cerebellar artery

internal carotid artery ophthalmic segment

internal carotid artery bifurcation segment

internal carotid artery posterior

communicating segment

SAH subarachnoid hemorrhage

ACT activated clotting time 


\section{Introduction}

Stent-assisted coiling (SAC) represents an effective and safe treatment option for complex and broad-based intracranial aneurysms. Even though several innovative endovascular concepts such as extra- or intra-aneurysmal flow diverters or bifurcation stents were established during the last decade, SAC remains one of the most frequently applied techniques for wide-necked aneurysms [1-5]. The first stent intended for the treatment of wide-necked aneurysms approved by the FDA in 2002 was the Neuroform Microdelivery Stent System (Stryker, Kalamazoo, MI, USA) [6]. In the meantime, several technical advancements resulted in the Neuroform EZ (Stryker, Kalamazoo, MI, USA) with an improved delivery system [7]. The latest technical advancement resulted in the Neuroform Atlas Stent (Stryker, Kalamazoo, MI, USA), a combined open- and closed-cell low-profile stent that is compatible with a 0.0165 in. microcatheter. Meanwhile, a variety of different low-profile self-expandable stents became available for the treatment of complex intracranial aneurysms [8]. Here we report our angiographic and clinical immediate and follow-up results, using the Neuroform Atlas Stent in the endovascular treatment of intracranial aneurysms.

\section{Material and methods}

\section{Selection criteria, patient population, and clinical presentation}

We retrospectively analyzed the institutional databases of two neurovascular institutions regarding SAC between July 2015 and December 2019. Aneurysms that were treated with the Neuroform Atlas Stent including adjunctive coiling on an intention-to-treat approach were included into further analysis. Procedures where the Neuroform Atlas Stent was used as a bail-out option (e.g., to fix a dislocated coil or a thrombus, $n=$ 7) or in combination with stents of other brands or different devices such as intra- or extra-aneurysmal flow diverters $(n=$ 11) were excluded from further analysis. Previous treatment of aneurysms was not an exclusion criterion.

The decision for endovascular treatment was made during an interdisciplinary neurovascular meeting at the participating centers, with at least one experienced neurosurgeon and neurointerventionalist. All patients were consented at least $24 \mathrm{~h}$ prior to the procedure except for acute subarachnoid hemorrhages. Procedures were performed by five experienced neurointerventionalists. Fixed inclusion or exclusion criteria were not defined, since this analysis represents a retrospective observation at the two participating centers. Arguments in favor for the use of Neuroform Atlas stent were broad-based morphology (dome to neck ratio $\leq 2$ ), a parent vessel diameter and anatomy suitable for the given dimensions of the
Neuroform Atlas Stent. Absolute criteria against the use of the Neuroform Atlas Stent were a fusiform morphology of the target aneurysm or a diameter of the parent artery below or above the recommended diameters (see description below). Broad-based bifurcation type aneurysms, originating eccentrically from the bifurcation, were a relative argument against the Neuroform Atlas Stent if a single-braided stent was determined sufficient to cover the entire neck due to the capability to bulge into the aneurysm. An isolated origin of an efferent parent artery from the aneurysm sac was a further argument against the Neuroform Atlas Stent. In cases of acutely ruptured aneurysms, vasospasm was an absolute argument against SAC.

The following variables were retrospectively recorded: clinical and demographic patient data according to the modified Rankin scale (mRS); technical success (including successful navigation, deployment and opening of the Neuroform Atlas stent); pre- and postprocedural angiographic data including aneurysm location, diameters of the target aneurysm (dome/neck), diameters of the parent artery proximal, and distal to the aneurysm origin; peri- and postprocedural complications; postprocedural occlusion rates according to the Raymond-Roy Occlusion Classification (RROC); and angiographic and clinical follow-up results according to the RROC and mRS. The initial and follow-up occlusion rate was determined by the operator and a second member of the neurovascular team, who did not perform the procedure. In cases of inconsistency concerning the final result, a decision was made by consensus.

We tried to identify factors likely to influence the occurrence of early reperfusion or the need for re-treatment. Therefore a separated subgroup analysis regarding aneurysm location, size (dome and neck width), previous treatment, and the treatment technique used for SAC (jailed microcatheter, re-cathetrization of the stent within the same or an additional procedure) was performed using the two-sample Wilcoxon rank-sum and the Fisher's Exact test ( $p$ values below 0.05 were defined as statistically significant).

\section{Neuroform Atlas Stent}

The Neuroform Atlas Stent is a self-expanding open- and closed-cell laser cut nitinol stent. While proximally the stent consists of closed cells in order to facilitate re-catheterization with a microcatheter, the distal part consists of an open-cell structure, which on the one hand improves a proper apposition to the vessel wall and on the other increases the stability within the distal landing zone of the artery. However, the open-cell design impedes the possibility to re-sheath the stent. The Neuroform Atlas stent is intended for vessel diameters from 2.0 to $4.5 \mathrm{~mm}$ in diameter. (available stent diameters 3.0, 4.0 and $4.5 \mathrm{~mm}$ with $15,21,24$ and $30 \mathrm{~mm}$ in length). Three 
radiopaque markers at the proximal and distal end guarantee the visualization of the stent within the artery, while the stent struts themselves are not visible under fluoroscopy. The design of the Neuroform Atlas stent allows for a compatibility with a 0.0165 in. microcatheter. After placement of the stent a mild foreshortening of 2.9-6.3 mm must be kept in mind depending on vessel size. A detailed description of the technical properties of the Neuroform Atlas Stent is given in the literature [9].

\section{Endovascular procedure}

All procedures were performed using a coaxial 8/6F guiding/ intermediate catheter or a $6 \mathrm{~F}$ guiding catheter alone in combination with a short $8 \mathrm{~F}$ vascular sheath. One microcatheter was placed distal to the aneurysm at the desired position of the stent (distal landing zone). An additional microcatheter was placed inside the aneurysm (jailing technique) in exceptional cases with an anticipated difficulty to catheterize the aneurysm after placement of the stent. The decision hereto was made by the operator. In cases where a second microcatheter was placed within the aneurysm sac before the stent opening, coiling proceeded the stent placement. In the majority of cases, a cautious attempt to re-cross the stent was made with the same microcatheter and the aneurysm was coiled. In cases of difficulties to catheterize the recently implanted stent, the procedure was terminated in order to avoid dislocation of the stent due to the microwire/microcatheter manipulations. The definitive occlusion of the aneurysm was carried out 6 weeks later in order to give time for an endothelialisation and with this stabilization of the stent.

\section{Anticoagulation/antiplatelet regimen}

All patients except those with ruptured aneurysms received a dual antiplatelet medication with $100 \mathrm{mg}$ acetylsalicylic acid (ASA) and $75 \mathrm{mg}$ Clopidogrel daily, 5 days prior to the scheduled treatment including an initial loading dose of $500 \mathrm{mg}$ ASA and $600 \mathrm{mg}$ Clopidogrel. Clopidogrel was continued for at least 3 months followed by life-long continuation of ASA. The efficacy was tested by an impedance aggregometry testing method (Multiplate ${ }^{\circledR}$, Roche GmbH, Germany). Values below 30 units in the ASPI test (arachidonic acid), which indicates the sensitivity for ASA and IIb/IIIa antagonists tested by activation of arachidonic acid converted by cyclooxygenase, indicated a sufficient effect of ASA. Correspondingly values below 45 units in the ADP test (adenosine diphosphate) that indicates sensitivity for Clopidogrel and IIb/IIIa antagonists tested by activation of ADP demonstrated a regular response to Clopidogrel.

Non-responders to Clopidogrel (indicated by an ADP test above 45 units) were changed to a loading dose of $180 \mathrm{mg}$ Ticagrelor followed by $90 \mathrm{mg}$ twice daily. The Multiplate ${ }^{\circledR}$ test was repeated until a sufficient platelet inhibition was clearly ascertained.

Eptifibatide was administered intravenously with a bolus followed by continuous intravenous application for at least $12 \mathrm{~h}$, bridged with a loading dose of $500 \mathrm{mg}$ ASA and $600 \mathrm{mg}$ Clopidogrel in cases of acutely ruptured aneurysms, where SAC was considered necessary. A bolus of 5000 units of heparin was given at the beginning of each procedure. The activating clotting time (ACT) was not monitored.

In cases of acute stent thrombosis glycoprotein IIb/IIIa inhibitors were administered on an individual decision.

\section{Follow-up schedule}

Angiographic follow-up examinations were scheduled between 3 and 6 months and 12 months following treatment with the Neuroform Atlas Stent according to the institutional standards. The angiographic results were independently analyzed by two interventional neuroradiologists. The clinical status of each patient was measured by a clinical team member according to the mRS.

\section{Ethical statement}

The study has been performed in accordance with the ethical standards laid down in the 1964 Declaration of Helsinki and its later amendments. A separate ethical approval was not required according to the local ethics committee guidelines (Ruhr-Universität Bochum, Germany) for this retrospective analysis without any additional examination or survey of the patients.

\section{Results}

\section{Patient population, aneurysm characteristics}

During the sample period, 446 aneurysms were treated by SAC at the two centers. In 112 patients (median age 56 years; range 29-90 years, 79 female) with 119 intracranial aneurysms the Neuroform Atlas Stent was applied. One hundred and eighth of $119(90.8 \%)$ were found incidentally, whereas $8 / 119(6.7 \%)$ were acutely ruptured (SAH within $48 \mathrm{~h}$ prior to treatment) and $3 / 119(2.5 \%)$ were symptomatic (emboli from the target aneurysm). Thirty-two of 119 (26.9\%) of all aneurysms were remnants of previously coiled or clipped aneurysms) and 6/119 (5.0\%) were incompletely occluded aneurysms after treatment with the WEB device (Woven Endobribge; Microvention, Tustin, CA, USA). One hundred and three of $119(86.6 \%)$ aneurysms were located in the anterior circulation compared with $16 / 119$ (13.4\%) in the posterior circulation. The median diameter of the aneurysm dome was 6.0 (range 1.0-20.0) with a median neck 
width of 5.0 (range 2.0-21.0). One hundred and fifteen of $119(96.6 \%)$ had a dome-to-neck ratio of $\leq 2$. Table 1 summarizes the patient and aneurysm characteristics.

\section{Immediate angiographic results}

Successful navigation and correct device placement of the Neuroform Atlas Stent across the aneurysm neck were as intended in all but one procedure (see case description below). A total of 133 devices were used in the entire cohort. A single stent was applied in 105/119 (88.2\%) of all cases, whereas in 14/119 cases (11.8\%) 2 Neuroform Atlas Stents were implanted in a $\mathrm{Y}, \mathrm{X}$,or $\mathrm{H}$ configuration. All stents were exclusively implanted with an Excelsior SL 10 microcatheter (Stryker, Kalamazoo, MI, USA).

Coiling of the target aneurysm was performed via a previously placed second microcatheter (jailed microcatheter) in $10 / 119(8.4 \%)$ of the cases, while in $90 / 119(75.6 \%)$ the Neuroform Atlas Stent was re-catheterized and crossed with the same microcatheter to coil the aneurysms within the same treatment session. A dislocation of the recently implanted stent following the re-catheterization was not observed. In 19/119 (16.0\%) re-catheterization and coil occlusion of the aneurysm were performed in a second procedure after cautious attempts to re-catheterize the Neuroform Atlas Stent failed. This second procedure was scheduled 6 weeks later. Of those 10 cases were aneurysms of the A com A.

Of the 8 aneurysms that were acutely ruptured, vasospasm was not observed during the index procedure. If necessary an external ventricle drainage was placed prior to the

Table 1 Summary of patient and aneurysm characteristics

\begin{tabular}{lll}
\hline & {$[\mathrm{N}]$} & {$[\%]$} \\
\hline Patients & 112 & 100 \\
Female & 79 & 70.5 \\
Male & 33 & 29.5 \\
Aneurysms & 119 & 100.0 \\
Anterior circulation & 103 & 86.6 \\
Posterior circulation & 16 & 13.4 \\
Incidental & 108 & 90.8 \\
Acute SAH & 8 & 6.7 \\
Symptomatic & 3 & 2.5 \\
& $\mathrm{Median}[\mathrm{mm}]$ & $\mathrm{Range}[\mathrm{mm}]$ \\
Aneurysm dome & 6.0 & $1.0-20$ \\
Aneurysm neck & 5.0 & $2.0-21.0$ \\
& {$[\mathrm{~N}]$} & {$[\%]$} \\
Successful stent placement & 118 & 99.2 \\
Thromboembolic complications & 2 & 1.7 \\
Hemorrhagic complications & 1 & 0.8 \\
\hline
\end{tabular}

procedure and the antiplatelet medication was administered as described $(N=4)$. Hemorrhagic complications related to this were not encountered.

The immediate angiographic result after SAC (whether performed in a single or two separated procedural steps) showed a complete occlusion (RROC I) in 99/119 (83.2\%) of all treated aneurysms; $15.1 \% 18 / 119(15.1 \%)$ had a residual neck (RROC II) and 1.7\% 2/119 (1.7\%) showed a residual opacification of the aneurysm.

\section{Follow-up results}

To date at least one follow-up angiography was carried out in 99/112 (88.4\%) patients with 105/119 (88.2\%) treated aneurysms after a median of 5 months (range 1-37 months). Of the remaining 13 patients, five died (four as consequence of the pre-existing SAH and one due to a cerebral hemorrhage (see case description below)). The additional eight patients were lost to follow-up.

A second follow-up angiography was available for $86 / 112$ (76.8\%) patients with 91/119 (76.5\%) treated aneurysms after a median of 12 months (range 2-34 months). Apart from the five patients described above, the remaining 21 were lost to follow-up to date.

Regarding the first and second follow-up angiography complete occlusion (RROC I) was observed in 79/105 (75.2\%) and in 74/91 cases (81.3\%).

In the first follow-up examination, seven out of 105 cases showed a mild and clinically silent in-stent stenosis (6.7\%).

No additional cases of intimal hyperplasia or in-stent stenosis were observed within the 12 months follow-up interval.

Tables 2, 3, 4 summarizes the immediate and follow-up angiographic results in detail, including the occlusion grade changes related to the initial angiographic result.

As shown in Table 3, 16 of the 105 (15.2\%) aneurysms showed an early reperfusion at the first follow-up angiography. To date a second treatment was carried out in 16/119 (13.4\%).

The separated analysis showed that previously treated and larger aneurysms were significantly more frequent in the two subgroups, while aneurysm location had no statistically

Table 2 Summary of immediate and follow-up results according to the RROC

\begin{tabular}{lllllll}
\hline & $\begin{array}{l}\text { Immediate } \\
{[\mathrm{n}]}\end{array}$ & $\begin{array}{l}\text { Ratio } \\
{[\%]}\end{array}$ & $\begin{array}{l}\text { FU1 } \\
{[\mathrm{n}]}\end{array}$ & $\begin{array}{l}\text { Ratio } \\
{[\%]}\end{array}$ & $\begin{array}{l}\text { FU2 } \\
{[\mathrm{n}]}\end{array}$ & $\begin{array}{l}\text { Ratio } \\
{[\%]}\end{array}$ \\
\hline Patients & 112 & 100 & 99 & 88.4 & 86 & 76.8 \\
Aneurysms & 119 & 100 & 105 & 88.3 & 91 & 76.5 \\
RROC1 & 99 & 83.2 & 79 & 75.2 & 74 & 81.3 \\
RROC2 & 18 & 15.1 & 25 & 23.8 & 16 & 17.6 \\
RROC3 & 2 & 1.7 & 1 & 1.0 & 1 & 1.1 \\
\hline
\end{tabular}


Table 3 Summary of short-term occlusion grade shifts (initial result to $\mathrm{F} / \mathrm{U}$ 1)

\begin{tabular}{lll}
\hline FU 1 & {$[n]$} & Ratio [\%] \\
\hline Total & 105 & 100 \\
Unchanged & 81 & 77.1 \\
Reperfusion & 16 & 15.2 \\
Improved occlusion & 8 & 7.7 \\
\hline
\end{tabular}

significant influence on the occurrence of early reperfusion or the need for a re-treatment.

The treatment strategy for SAC did not affect the occurrence of early reperfusion, while in the subgroup of retreated aneurysms, the proportion of those coiled within a second procedure was significantly higher.

\section{Illustrative cases (Figs. 1a-d, 2a-e)}

\section{Clinical results and complications}

In one case of a recurrent anterior communicating artery (A com A) aneurysm, previously treated by SAC with the Neuroform Atlas Stent placed across the aneurysm from the right $\mathrm{A} 2$ to the left $\mathrm{A} 1$ segment, the operator placed a second stent within the left ACA across the aneurysm with its proximal markers ending at the previously implanted right-sided stent in a $\mathrm{Y}$ configuration. A dislocation of coil loops into the lumen of the A com A occurred, which was probably the result of an unrecognized gap between the two stents. The coil loops were fixed with a further stent placed across the gap at the A com A from the left A2 to the right A1 segment without technical problems. The patient remained clinically asymptomatic following the described procedure.

Two thromboembolic complications occurred. In one patient a thrombus formation within the Neuroform Atlas stent occurred during re-treatment of an MCA bifurcation aneurysm, which was treated successfully by a weight adapted intravenous bolus of tirofiban according to an individual decision of the operator. The patient remained without a clinical sequel. Another patient with a right-sided MCA bifurcation aneurysm suffered from an acute in-stent thrombosis with a subsequent occlusion of the M2 segment 6 months after the initial procedure. At that time the patient had been on a monotherapy of $100 \mathrm{mg}$ of ASA for 6 weeks. The occlusion resolved following intravenous thrombolysis (IVT). The patient deteriorated clinically to a mRS score of 3 at discharge.

Table 4 Summary of long-term occlusion grade shifts (initial result to $\mathrm{F} / \mathrm{U} 1$ )

\begin{tabular}{lll}
\hline FU 2 & {$[n]$} & Ratio [\%] \\
\hline Total & 91 & 100 \\
Unchanged & 78 & 85.7 \\
Reperfusion & 4 & 4.4 \\
Improved occlusion & 9 & 9.9 \\
\hline
\end{tabular}

One fatal cerebral hemorrhage occurred 2 days after treatment of a MCA bifurcation aneurysm. In this patient, an acute occlusion of the right femoral artery was successfully treated by implantation of a self-expandable stent with $4 \mathrm{mg}$ of recombinant tissue plasminogen activator given intra-arterially during this procedure. The multifocal right-sided intracerebral hemorrhage that occurred directly after treatment of the femoral occlusion was potentially induced by the combination of intra-arterial recombinant tissue plasminogen activator and the pre-existing dual antiplatelet therapy. Four patients died as consequence of the underlying SAH.

The procedure-related morbidity and mortality in the entire series are $1 / 112(0.9 \%)$ and $1 / 112(0.9 \%)$, respectively.

\section{Discussion}

The low-profile design of the Neuroform Atlas stent allows for the implantation via a 0.0165 in. microcatheter with a high technical success rate which is demonstrated by our experience with only one case of suboptimal positioning. These findings are in line with those reported from other publications [10-14]. In $75.6 \%$ of our cases, stent implantation and coil occlusion of the aneurysm were performed using the same microcatheter, which indicates a mostly trouble-free possibility to cross the struts. The option to perform SAC with only one microcatheter might lower the risk of thromboembolic complications especially in small distally located arteries. This strategy refers to our standard approach. In cases of an anticipated difficulty to re-cross the stent within the same session (e.g., curved anatomy of the stented artery) a second jailed microcatheter was used. This was done at the operator's individual decision. In 19 cases, where a re-catheterization with coiling was performed in a second procedure, the operator experienced unexpected difficulties to re-cross the recently implanted stent within the initial procedure. Our concept is to schedule a second straightforward procedure rather than to perform repetitive attempts to re-cross the stent, which might increase the risk of complications. This staged strategy requires a second procedure with an additional risk of complications, while the aneurysm is left perfused for 6 weeks, which in contrast to a jailed microcatheter represents a disadvantage of this approach and might serve as an argument for the jailing concept [15]. Interestingly 10 of the 19 cases where the re-catheterization of the stent failed were A com A aneurysms. This might be explained by the higher angles of the parent arteries in A com A aneurysms compared with typical side-wall aneurysms.

Our results confirm the safety and efficacy in the treatment of wide-neck intracranial aneurysms using SAC with the Neuroform Atlas Stent. The initial complete occlusion rate of $83.3 \%$ with comparable results during the mid- and longterm follow-up period is similar to previously published occlusion rates achieved with the Neuroform Atlas Stent. A 
Fig. 1 a Previously ruptured partly coiled A com A aneurysm, injection of the right ICA, small A1 segment, digital subtraction angiography, right- anterior oblique-caudal view. b Placement of a Neuroform Atlas Stent $(3.0 \times 24 \mathrm{~mm})$ from the left A2 to the left A1 segment, digital subtraction angiography, right- anterior oblique-caudal view. c After implantation of a second

Neuroform Atlas Stent

$(3.0 \times 24 \mathrm{~mm})$ from the right $\mathrm{A} 2$ to the left A1 segment (Y-Stenting), coiling of the aneurysm through a previously placed microcatheter via the right A1 segment, digital subtraction angiography, rightanterior oblique-caudal view. $\mathbf{d}$ Twelve months follow-up angiography showing near complete occlusion of the A com A aneurysm (RROC 2), digital subtraction angiography, and anteriorposterior caudal view
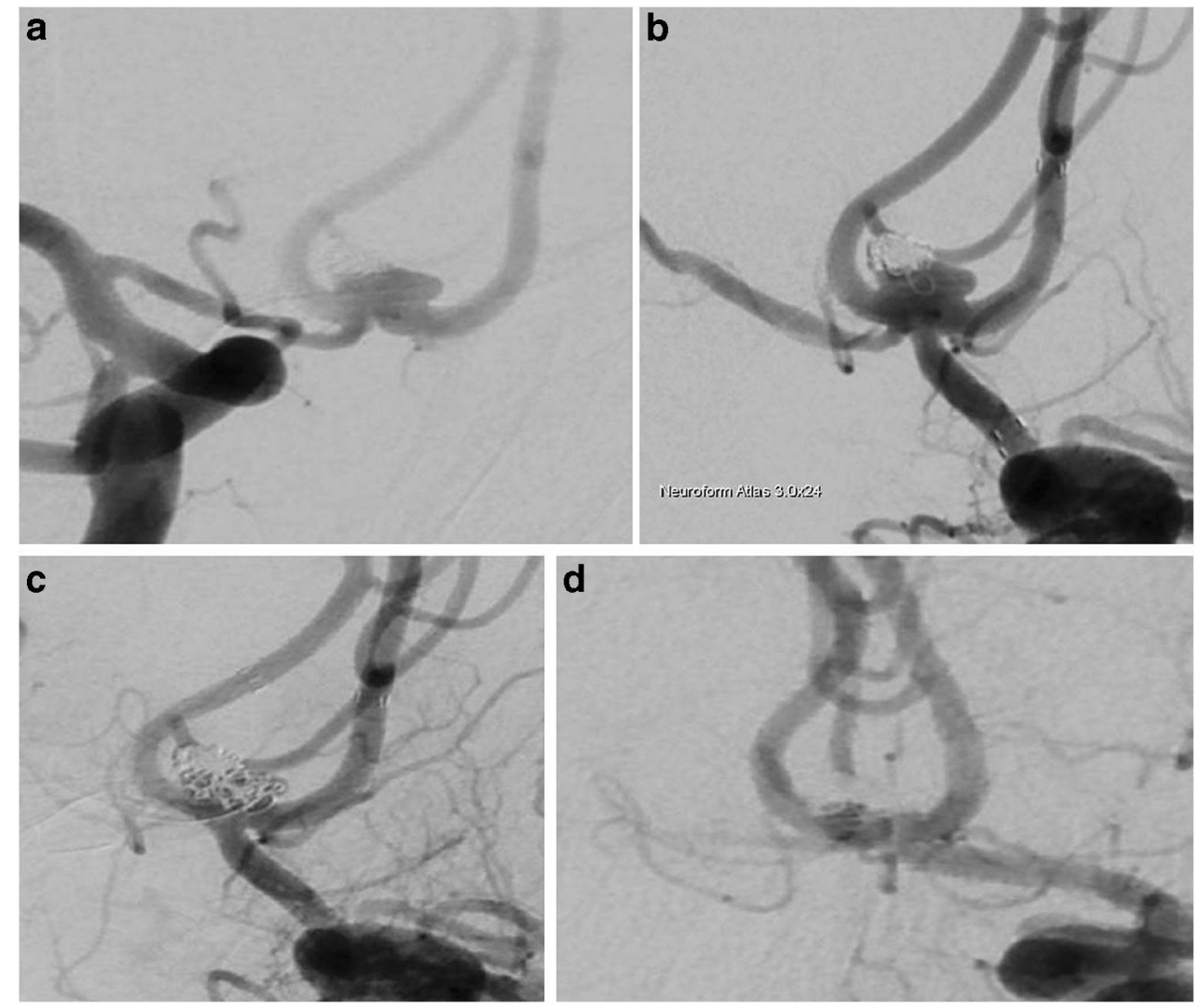

smaller series of 30 patients with 27 intracranial aneurysms revealed complete aneurysm occlusion in $92.6 \%$ after 12 months with a technical success rate of $100 \%$ [9]. A multicenter registry of 113 wide-necked aneurysms treated by Neuroform Atlas Stent-assisted coiling provided comparable results with a complete occlusion rate of $82.0 \%$ at 12 months and $100 \%$ technical success [11].

Djurdjevic et al. in their series of 101 intracranial aneurysms treated with Leo baby stent-assisted coiling (a braided low-profile stent) observed an initial complete and near complete occlusion rate of 24.7 and $43.0 \%$ [16]. In a meta-analysis of intracranial aneurysms treated with low-profile braided stents by Park et al., the initial complete and near complete occlusion rate was $87 \%$ [8]. These results are largely comparable to the results found in our study. However, a direct comparison of aneurysms treated with braided and laser cut stents remains inappropriate, since braided stents offer a mild flow-diverting effect, which might allow for an initial loose coiling in contrast to laser-cut stent-assisted coiling, where a dense coil packing is essential.

The safety of the Neuroform Atlas Stent regarding thromboembolic events appears to be high, a fact that is probably related to the lower radial force and the proper apposition to the vessel wall as a result of the open-cell design. We observed two thromboembolic complications with one being clinically relevant. This comparatively low rate is confirmed by other studies on the Neuroform Atlas Stent. Jankowitz et al., for example, in their series of 30 patients found one stroke ipsilateral to the treated aneurysm without a remaining neurological deficit in that patient [9]. The complication rate in the above mentioned meta-analysis of Park and al. was $12.4 \%$, while thromboembolic events occurred in $6.5 \%$ [8].

Some authors found a higher recanalization rate with open-cell stents $[10,17]$. One possible explanation might be found in a mild flow-diverting effect of braided stents due to their lower porosity. Furthermore, open-cell stents may show an increased cell size at the outer curve of the parent artery caused by a phenomenon called gator backing, which might lower the possible packing density of coils [18]. We found a progressive reperfusion in $15.2 \%$ of the cases at the 3 months follow-up angiography with a re-treatment rate of $13.4 \%$.

Our subgroup analysis of early reperfused and retreated aneurysms found a significantly higher rate of larger and previously treated aneurysms. This is not surprising since larger aneurysms are known to be more prone to reperfusion due to a compaction of coils. The factor "previous treatment" might indicate the complex cases that require repeated treatment steps in general.

In the subgroup of retreated aneurysms, the proportion of those coiled within a second procedure was significantly higher. This is most likely explained by the fact that these were cases were a re-catheterization of the recently implanted 
Fig. 2 a Broad-based aneurysm of the A com A, right anterioroblique and caudal view. $\mathbf{b}$ Implantation of a Neuroform Atlas Stent $(3.0 \times 24 \mathrm{~mm})$ from the right $\mathrm{A} 2$ to the right $\mathrm{A} 1$ segment, right anterior-oblique, and caudal view. $\mathbf{c}$ Re-catheterization of the stent with the Excelsior SL 10 microcatheter within the same session, right anterior-oblique and caudal view. d Coil occlusion of the aneurysm, right anterioroblique, and caudal view. e 12month follow-up angiography revealing stable occlusion of the aneurysm, right anterior-oblique, and caudal view
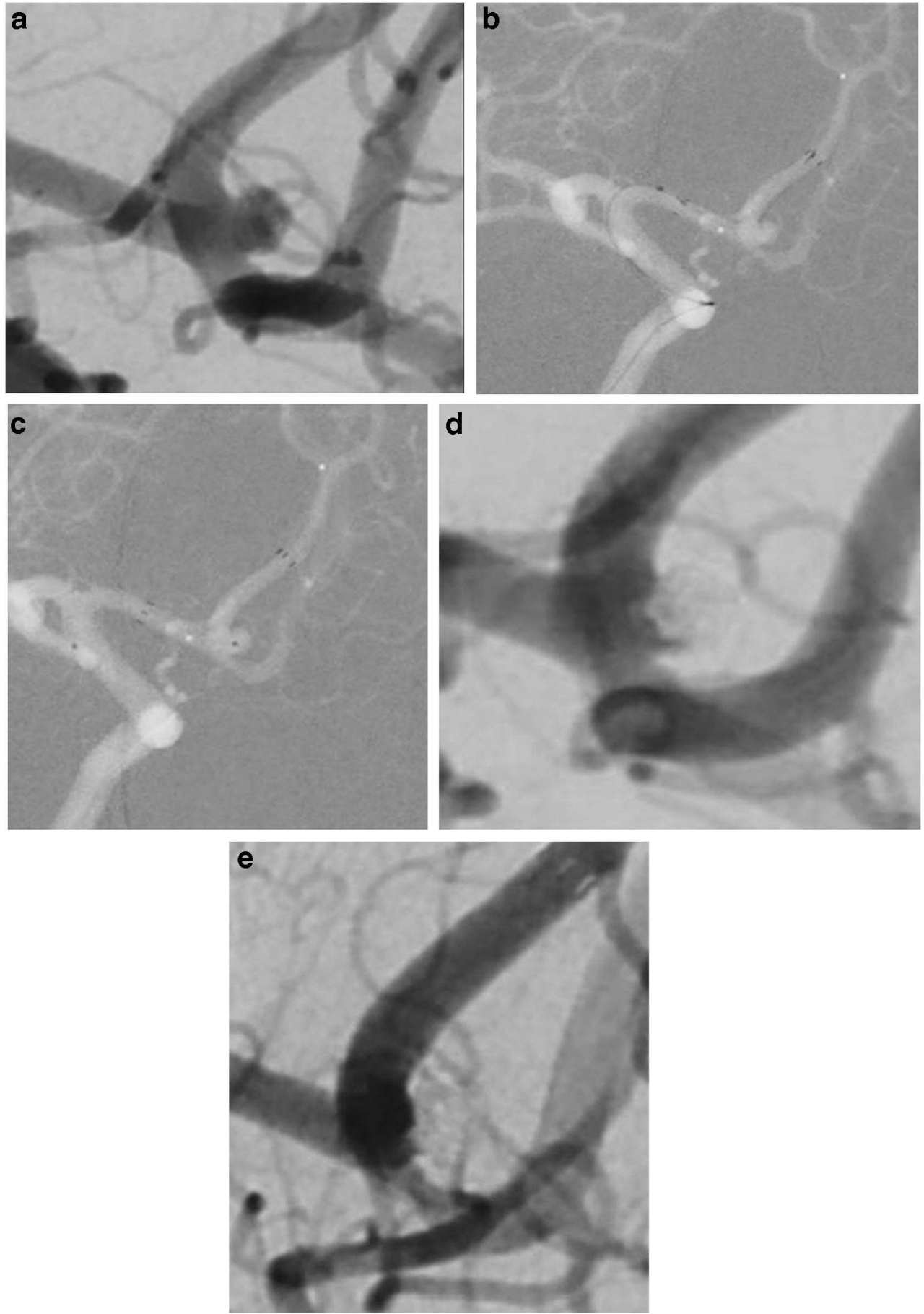

stent failed within in the initial procedure indicating a more complex situation (e.g., high grade angles of the parent arteries). Therefore in the second procedure, the position of the microcatheter might have been suboptimal which impeded a dense packing of the coils. Compared with those aneurysms treated with a jailed microcatheter, the potentially achievable packing density might be lower in "re-crossed" cases since the microcatheter and the final coils might more easily dislocate into the lumen of the stent. This might explain a more defensive strategy of the operator in re-crossed cases. These potential explanations remain speculation since the subgroups of reperfused and retreated aneurysm are too small for a conclusive multivariate analysis.

Goertz et al. in their series of 37 aneurysms treated with the Neuroform Atlas Stent observed a neck or aneurysm remnant in $19.2 \%$ with a re-treatment rate of $7.7 \%$, while a comparative analysis of intracranial aneurysms treated with either Neuroform Atlas or LVIS Jr. stent-assisted coiling showed 
greater obliteration rates and lower rates of in-stent stenosis in aneurysms treated with the Neuroform Atlas Stent [19, 20].

\section{Limitations}

Our study has several limitations. These are mainly related to retrospective character of the study without predefined inclusion and exclusion criteria. The angiographic results are self-assessed without an independent core lab, and the lack of a control group impedes a direct comparison with alternative treatment options.

\section{Conclusions}

Our two-center analysis demonstrates the safety and efficacy of SAC using the Neuroform Atlas Stent in the treatment of broad-based aneurysms. The clinical and angiographic results of our study are in line with those previously published. Prospective comparative studies might help to identify the value of SAC using the Neuroform Atlas stent among the growing treatment options for broad-based aneurysms.

Funding No funding was received for this study.

\section{Compliance with ethical standards}

Conflict of interest The authors declare that they have no conflict of interest related to this study.

Author 1 none.

Author 2 none.

Author 3 agreement with Stryker for proctoring services and consulting. Author 4 agreement with Stryker for proctoring services and consulting. Author 5 agreement with Stryker for proctoring services and consulting. Author 6 agreement with Stryker for proctoring services and consulting.

Ethical approval All procedures performed in the studies involving human participants were in accordance with the ethical standards of the institutional and/or national research committee and with the 1964 Helsinki Declaration and its later amendments or comparable ethical standards.

Informed consent A separate informed consent from each patient before inclusion in this study was not required due to the retrospective design of the study.

\section{References}

1. Cheung NK, Chiu AHY, Cheung A, Wenderoth JD (2018) Long term follow-up of bifurcation aneurysms treated with braided stent assisted coiling and complex T- and $\mathrm{Y}$ stent constructs. J Neurointervent Surg 10:560-565

2. Teramoto S, Oishi H, Arai H (2019) Comparative analysis of longterm effect of stent-assisted coiling in unruptured sidewall-type and terminal-type aneurysms. World Neurosurg 126:e753-e757

3. Mine B, Monnet T, Vazguez-Suarez JC, Iosif C, Lubicz B (2018) Comparison of stents used for endovascular treatment of intracranial aneurysms. Expert Rec Med Devices 15:793-805
4. Velasco Gonzalez A, Stracke O, Nordmeyer H, Heddier M, Saleme S, Sauerland C, Berkemeyer S, Buerke B, Heindel W, Chapot R (2018) Low rates of recanalization for wide-necked aneurysms treated with stenting after balloon-assisted coiling: combination of techniques delivers stable and improved results during follow-up. Neuroradiology 60:1223-1230

5. Wang J, Wargas J, Spiotta A, Chaudry I, Turner RD, Lena J, Turk A (2018) Stent-assisted coiling of cerebral aneurysms: a singlecenter clinical and angiographic analysis. J Neurointervent Surg 10:687-692

6. Henkes H, Bose A, Felber S, Miloslavski E, Berg-Dammer E, Kühne D (2002) Endovascular coil occlusion of intracranial aneurysms assisted by a novel self-expandable Nitinol microstent (Neuroform). Interv Neuroradiol 8:107-119

7. Mangubat EZ, Johnson AK, Keigher KM, Lopes DK (2012) Initial experience with Neuroform EZ in the treatment of wide-neck cerebral aneurysms. Neurointervention 7:34-39

8. Park SY, Oh JS, Oh HJ, Yoon MS, Bae HG (2017) Safety and efficacy of low-profile, self-expandable stents for the treatment of intracranial aneurysms: initial and midterm results-a systematic review and meta-analysis. Intervent Neurol 6:170-182

9. Jankowitz BT, Hanel R, Jadav AP, Loy DN, Frei D, Siddiqui A, Puri AS, Khaldi A, Turk AS, Malek AM, Sauvageau E, Hetts SW, Zaidat OO (2019) Neuroform atlas stent system for the treatment of intracranial aneurysm: primary results of the atlas humanitarian device exemption cohort. J NeuroIntervent Surg 11:801-806

10. Cay F, Peker A, Arat A (2018) Stent-assisted coiling of cerebral aneurysms with the Neuroform Atlas stent. Interv Neuroradiol 24: 263-269

11. Caragliano A, Rosario P, Pitrone A, Limbucci N, Nappini S, Ruggerio M, Visconti E, Alexandre A, Menozzi R, Lauretti D, Cavasin N, Alibrandi A, Tessitore A, Longo M, Vinci SL (2019) The low-profile Neuroform Atlas stent in the treatment of widenecked intracranial aneurysms-immediate and midterm results: an Italian multicenter registry. J Neuroradiol 47:421-427

12. Ulfert C, Pham M, Sonnberger M, Amaya F, Trenkler J, Bendszus M, Möhlenbruch MA (2018) The Neuroform Atlas stent to assist coil embolization of intracranial aneurysms: a multicenter experience. J Neurointervent Surg 10:1192-1196

13. Tsai JP, Hardman J, Moore NZ, Hussain MS, Bain MD, Rasmussen PA, Masaryk TJ, Elgabaly MH, Sheikhi L, Toth G (2019) Early post-humanitarian device exemption experience with the Neuroform Atlas stent. J Neurointervent Surg 11:1141-1144

14. Quintana EM, Valdes PV, Deza EM, Garcia AG, Rodriguez MC, Perez JMJ, Chaviano J, Morales JCG, Batista KP, Garcia FA (2019) Initial experience and one-year follow-up with Neuroform Atlas stent system for the treatment of brain aneurysms. Interv Neuroradiol 25:521-529

15. Geyik S, Yavuz K, YurttutanN SI, Cekirge HS (2013) Stentassisted coiling in endovascular treatment of 500 consecutive cerebral aneurysms with long-term follow-up. AJNR Am J Neuroradiol 34:2157-2162

16. Djurdjevic T, Young V, Corkill R, Briley D, Küker W (2019) Treatment of broad-based intracranial aneurysms with low profile braided stents: a single center analysis of 101 patients. J Neurointervent Surg 11:591-597

17. King B, Vaziri S, Singla A, Fargen KM, Mocco J (2015) Clinical and angiographic outcomes after stent-assisted coiling of cerebral aneurysms with Enterprise and Neuroform stents: a comparative analysis of the literature. J Neurointervent Surg 7:905-909

18. Bendorf G, Claus B, Strother C, Chang J, Klucznik RP (2008) Increased cell opening and prolapse of struts of a neuroform stent in curved vasculature: value of angiographic computed tomography: technical case report. Neurosurgery 58:ONS-E380 discussion ONS-E380 
19. Goertz L, Dorn F, Siebert E, Herzberg M, Borggrefe J, Schlamann M, Krischek B, Stavrinou P, Mpotsaris A, Bohner G, Liebig T, Kabbasch C (2019) Safety and efficacy of the Neuroform Atlas for stent-assisted coiling of intracranial aneurysms: a multicenter experience. J Clin Neurosci 68:86-91
20. Gross BA, Ares WJ, Ducruet AF, Jadhav AP, Jovin TG, Jankowitz BT (2019) A clinical comparison of atlas and LVIS Jr stent-assisted aneurysm coiling. J Neurointervent Surg 11:171-174 